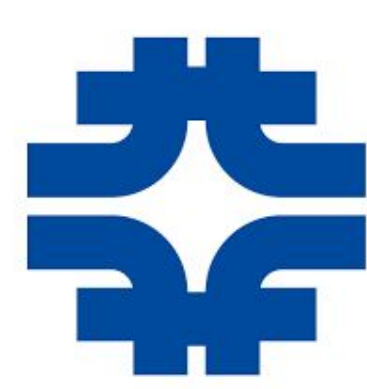

\title{
DES in 10 minutes
}

\section{DES in 7 minutes, Balrog in 3 minutes}

\author{
Megan Splettstoesser \\ Fermilab New Perspectives \\ June 18, 2018
}

This document was prepared by [DES Collaboration] using the resources of the Fermi National Accelerator Laboratory (Fermilab), a U.S. Department of Energy, Office of Science, HEP User Facility. Fermilab is managed by Fermi Research Alliance, LLC (FRA), acting under Contract No. DEAC02-07CH11359. 


\section{Dark Energy Survey (DES) Collaboration}

- $\sim 400$ collaborators, of which $\sim 70 \%$ are students and postdocs

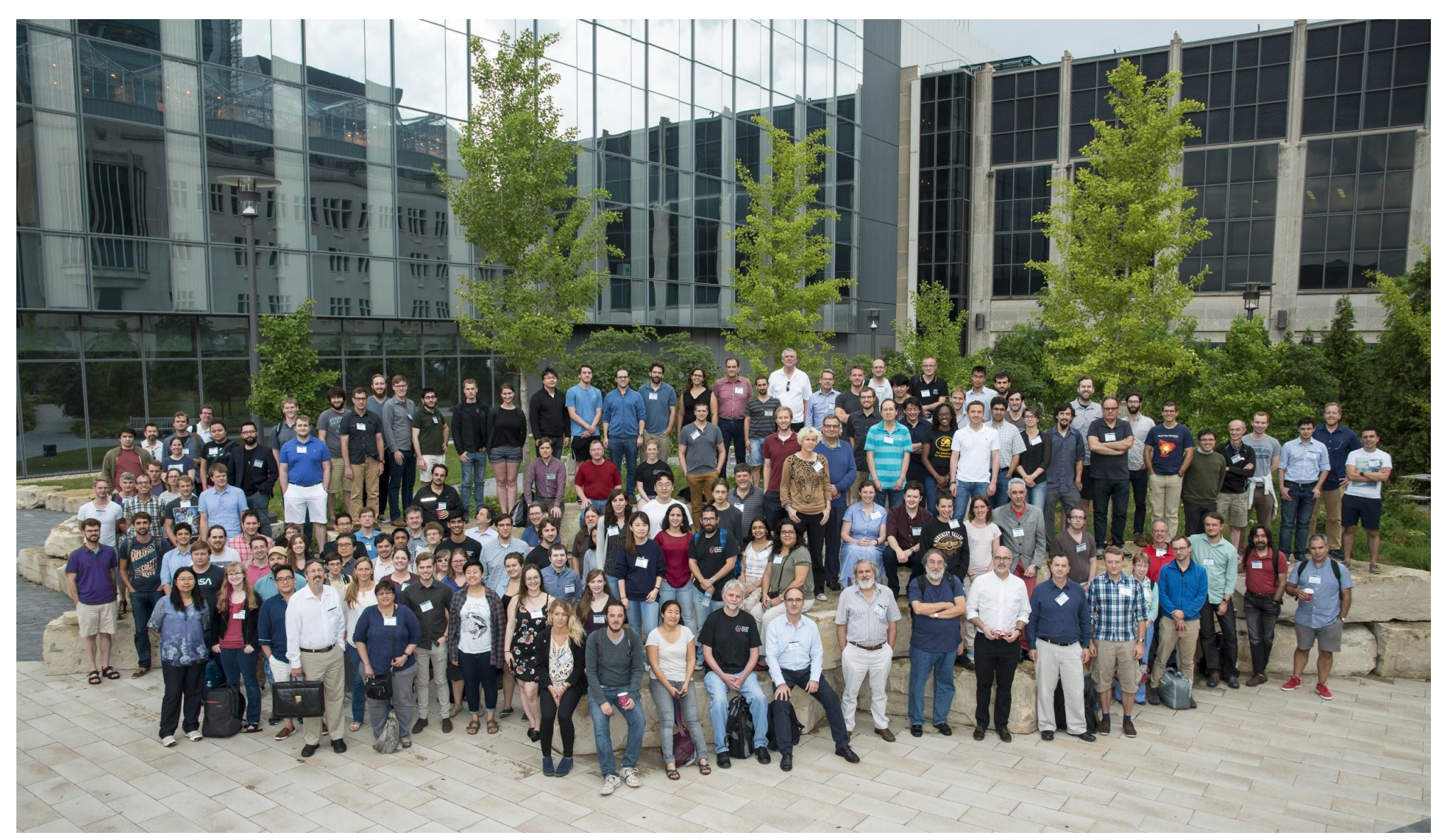




\section{Dark Energy Survey Member Institutions}

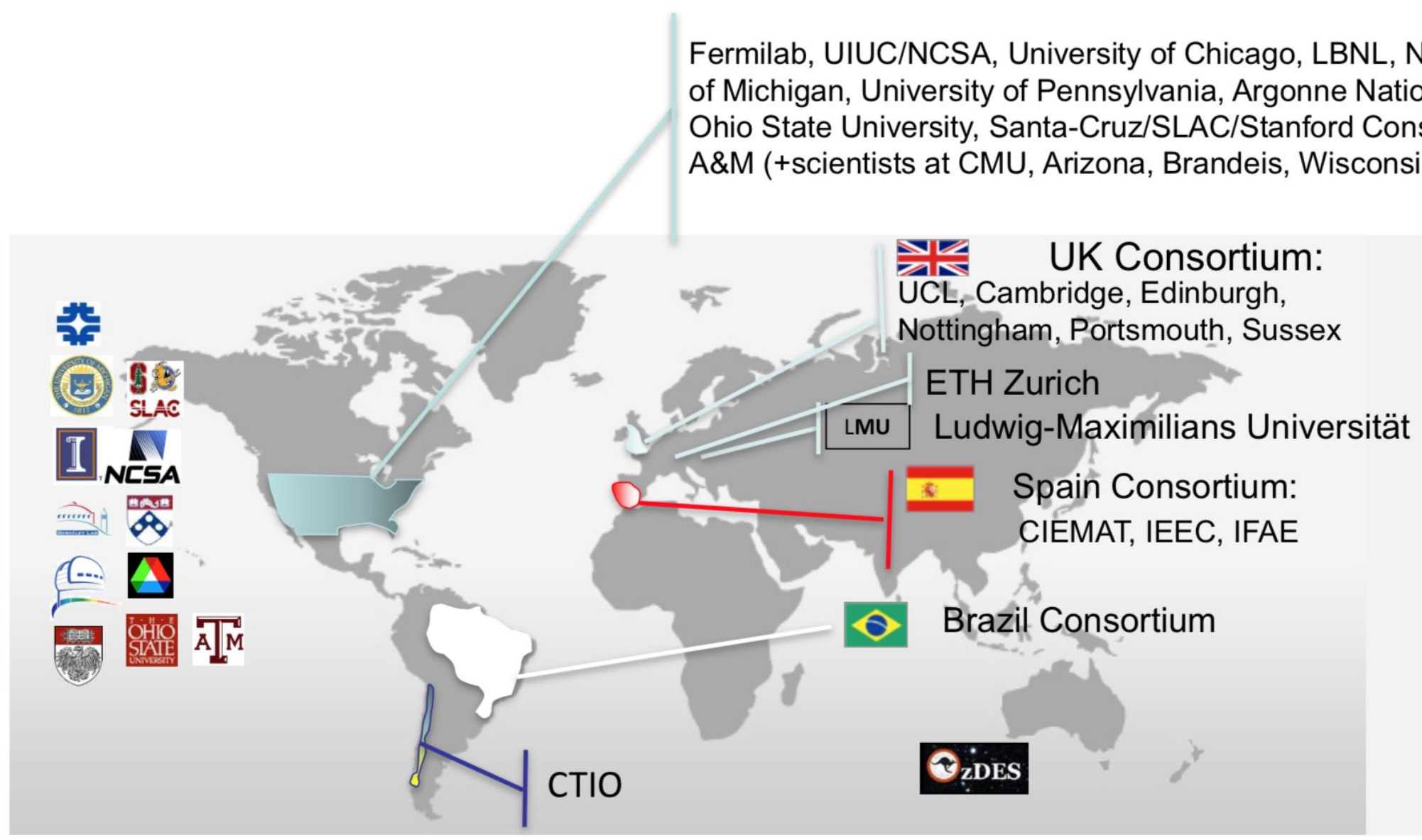




\section{Dark Energy Survey Science Goals}
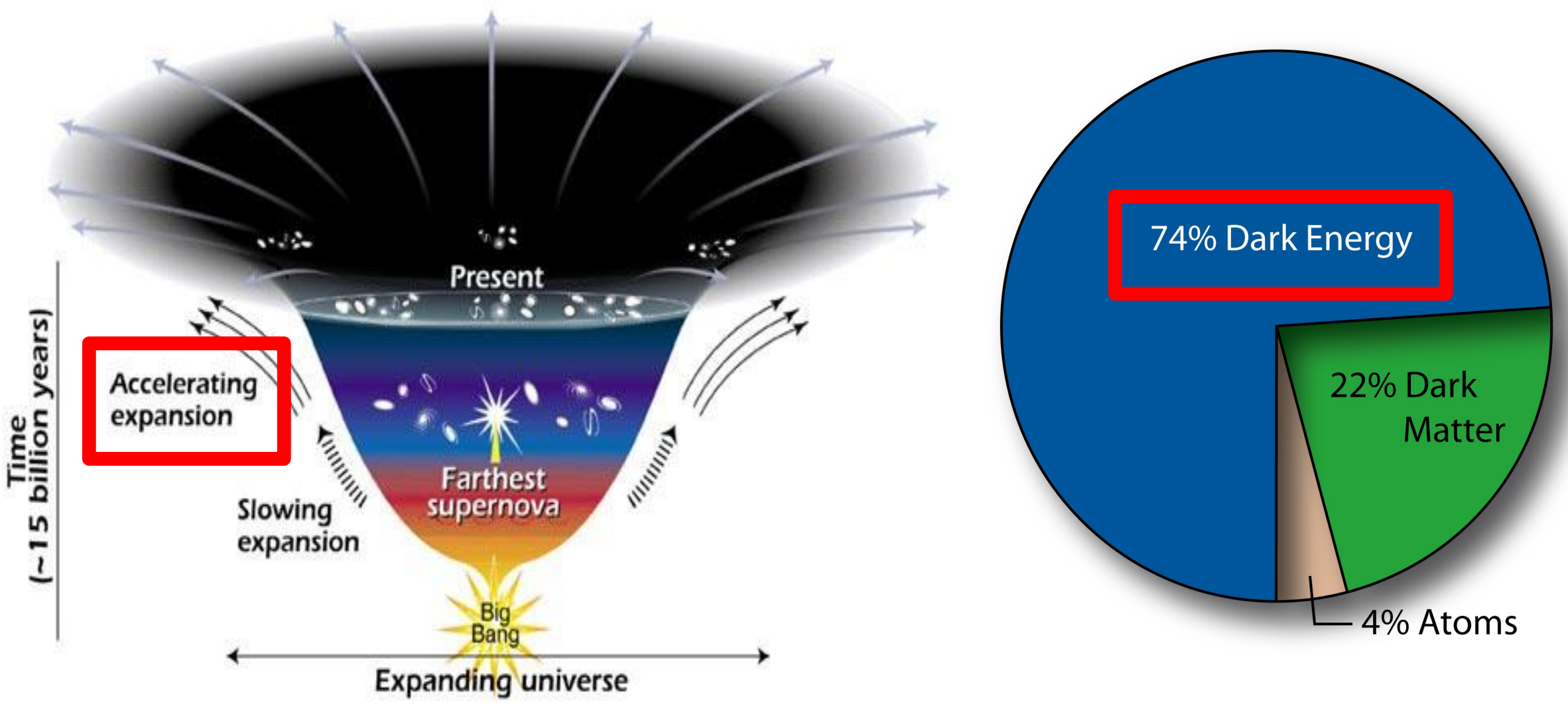


\section{Dark Energy Camera}
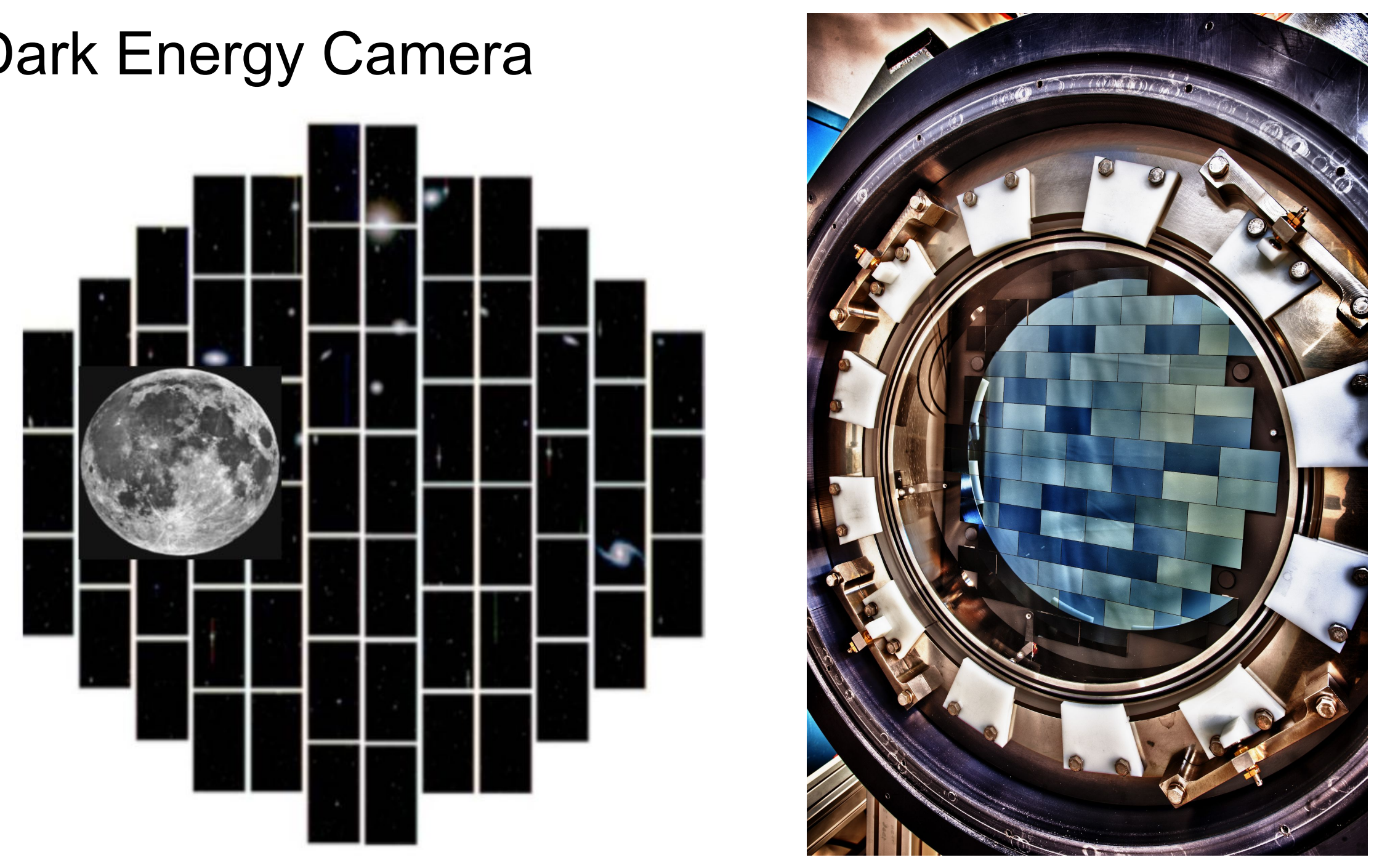

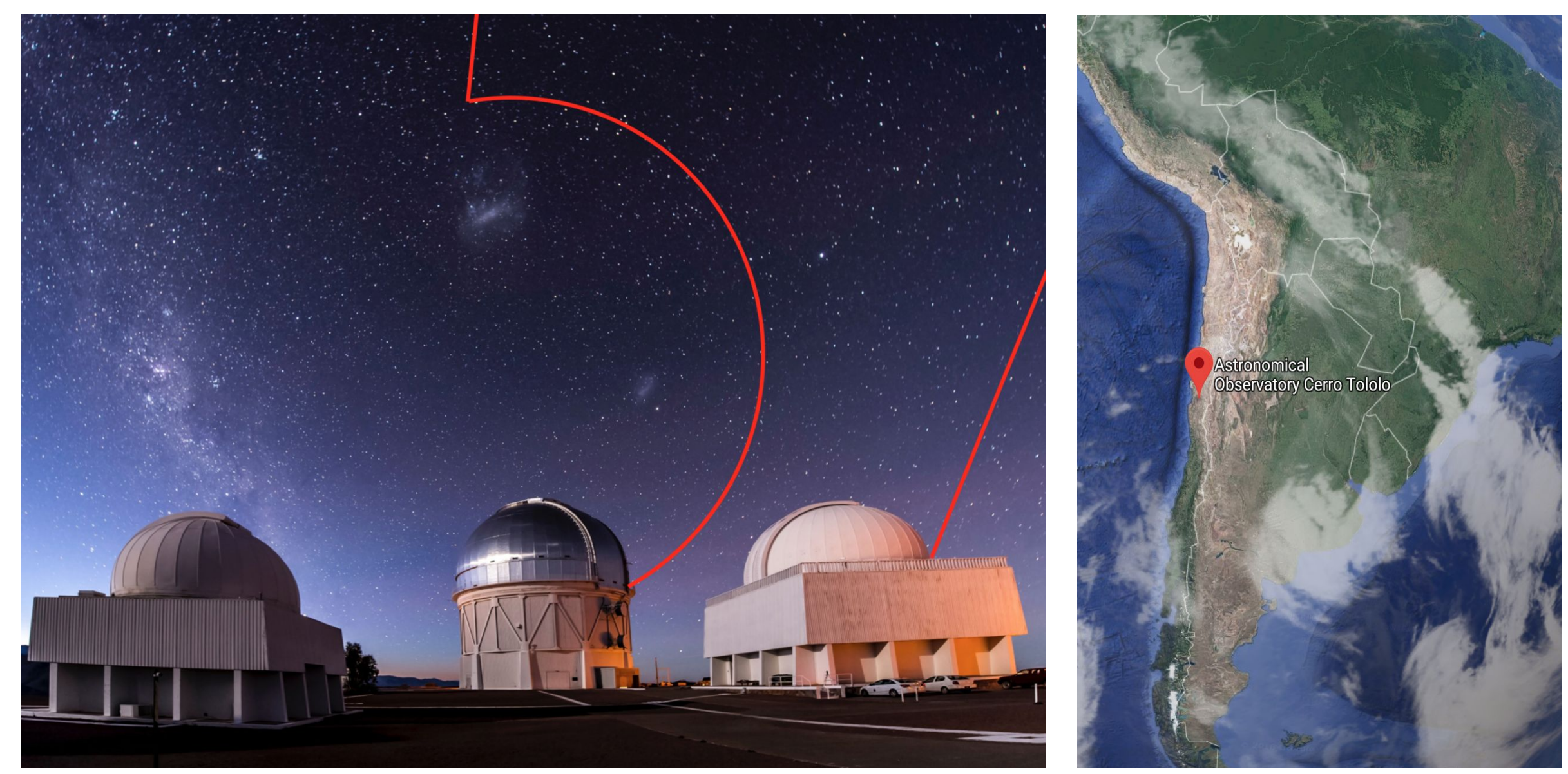


\section{DES Data Release 1: Galaxies}
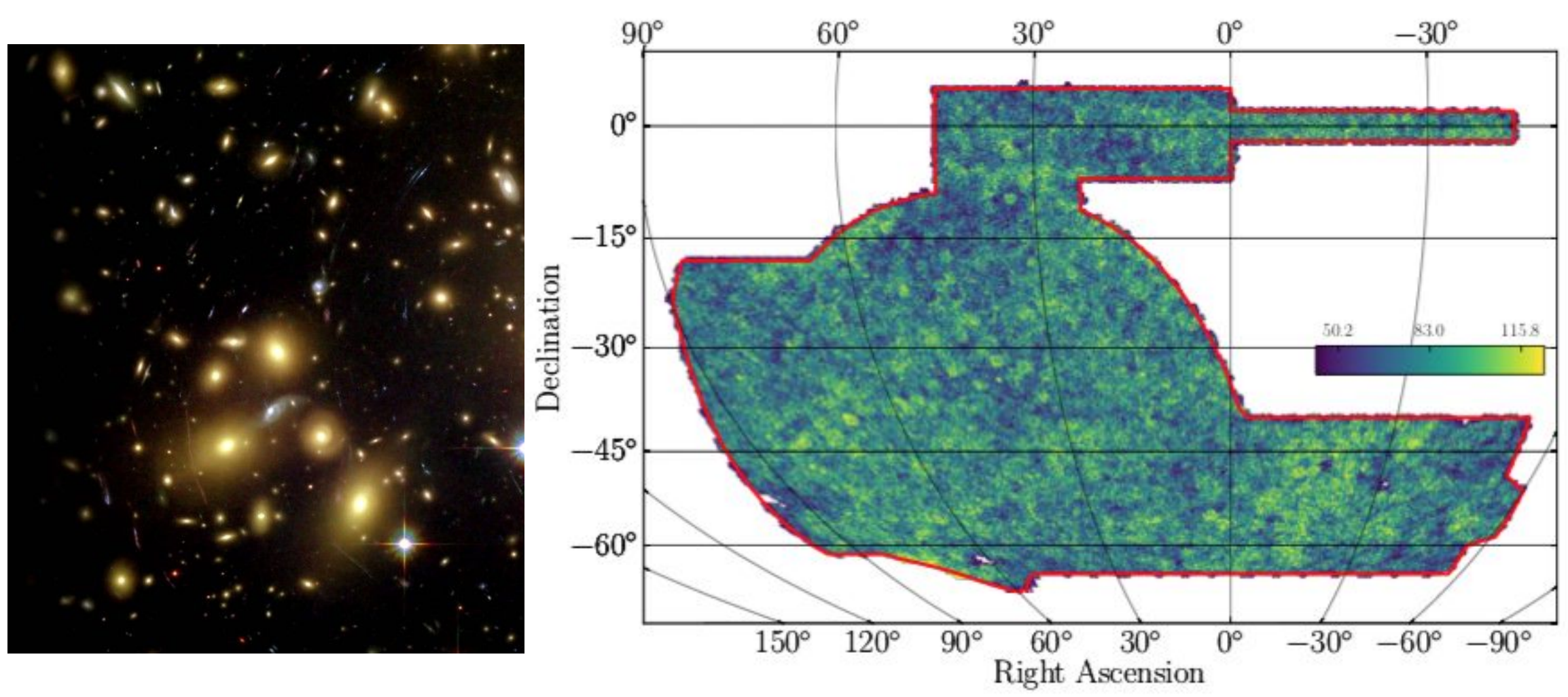

DES Collaboration [1] 


\section{DES Data Release 1: Stars}
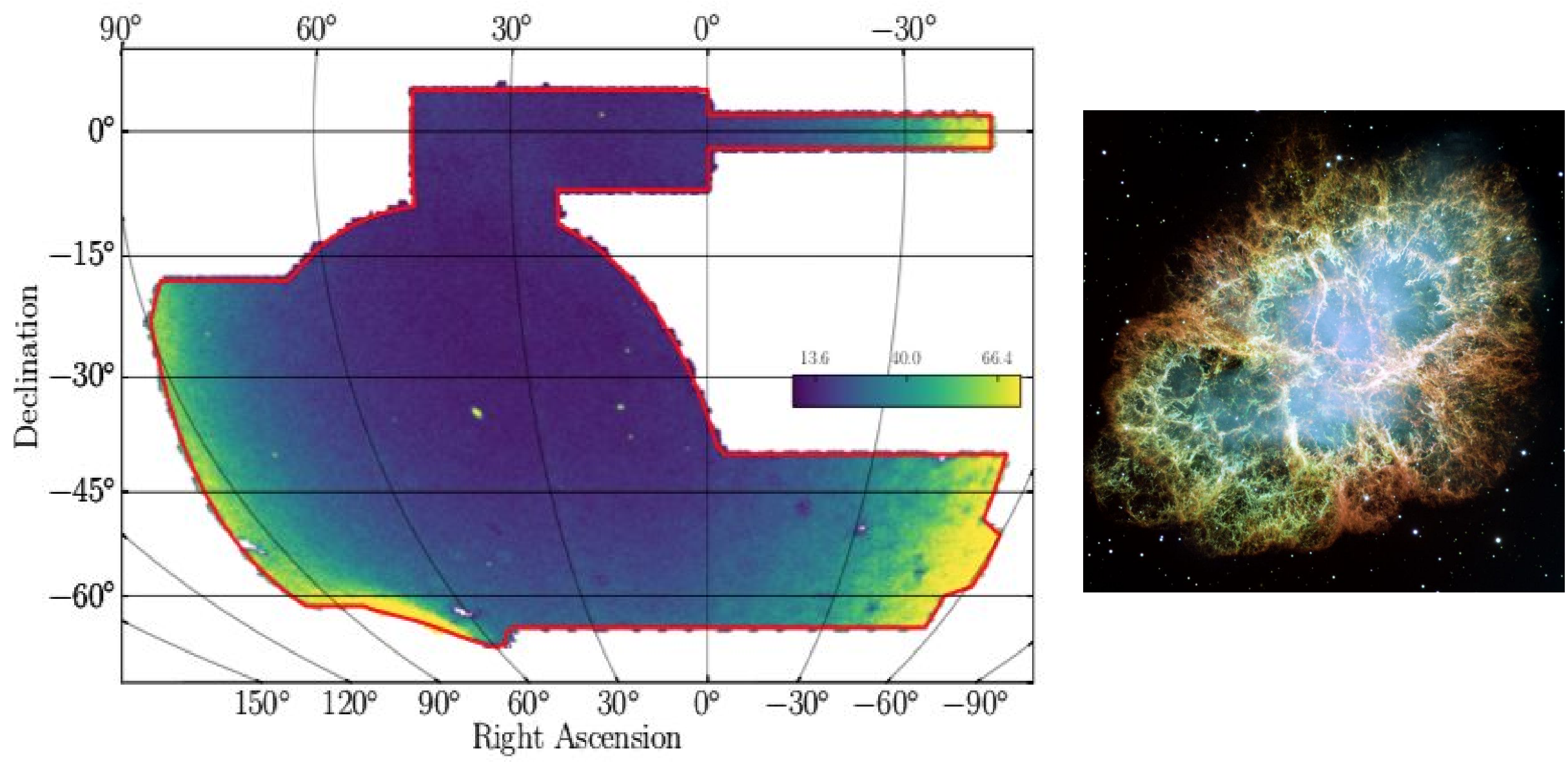

DES Collaboration [1] 


\section{DES DR1 discovered 11 stellar streams}

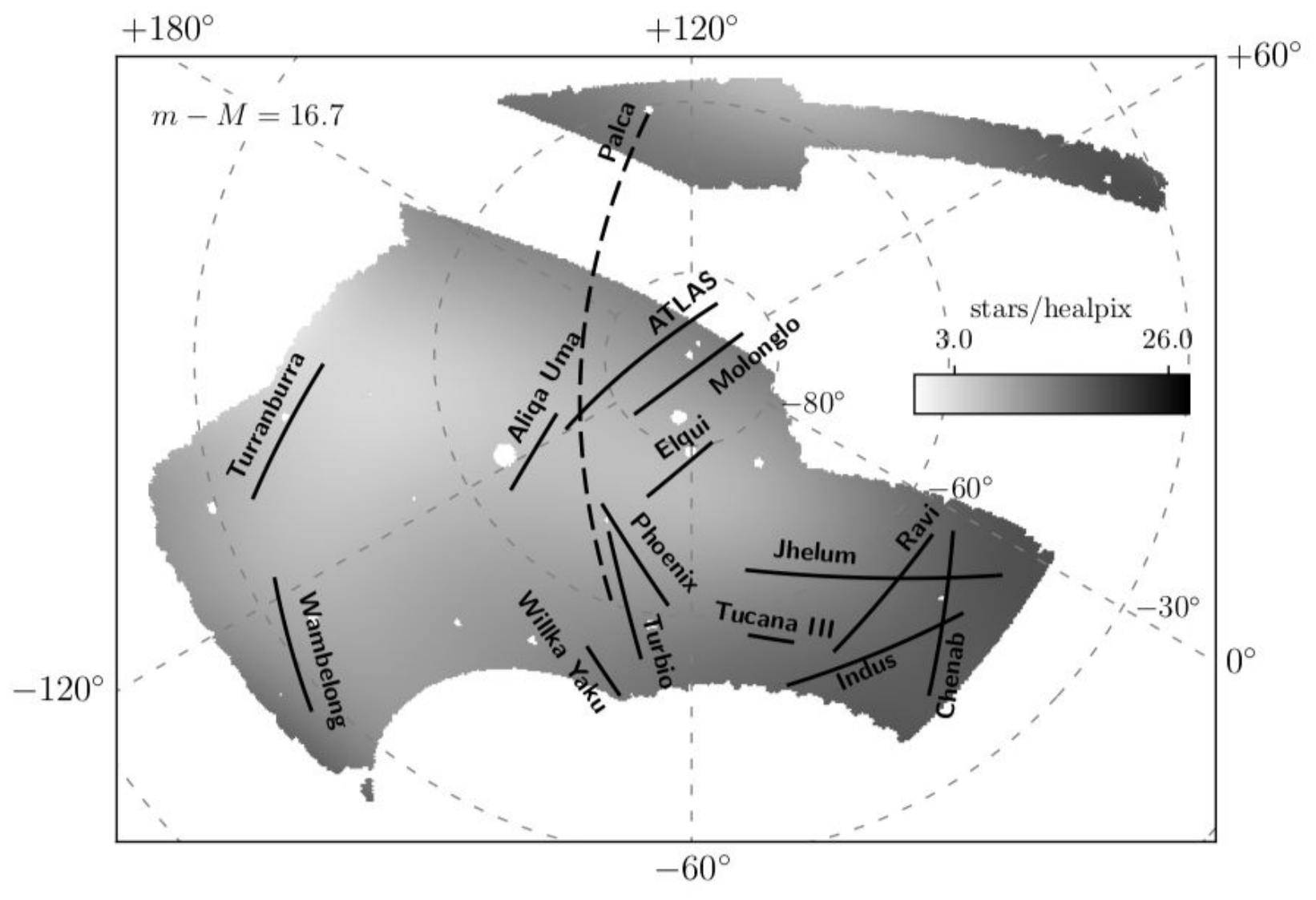

Shipp et al. [2] 
DES Y1 constraints on cosmological parameters
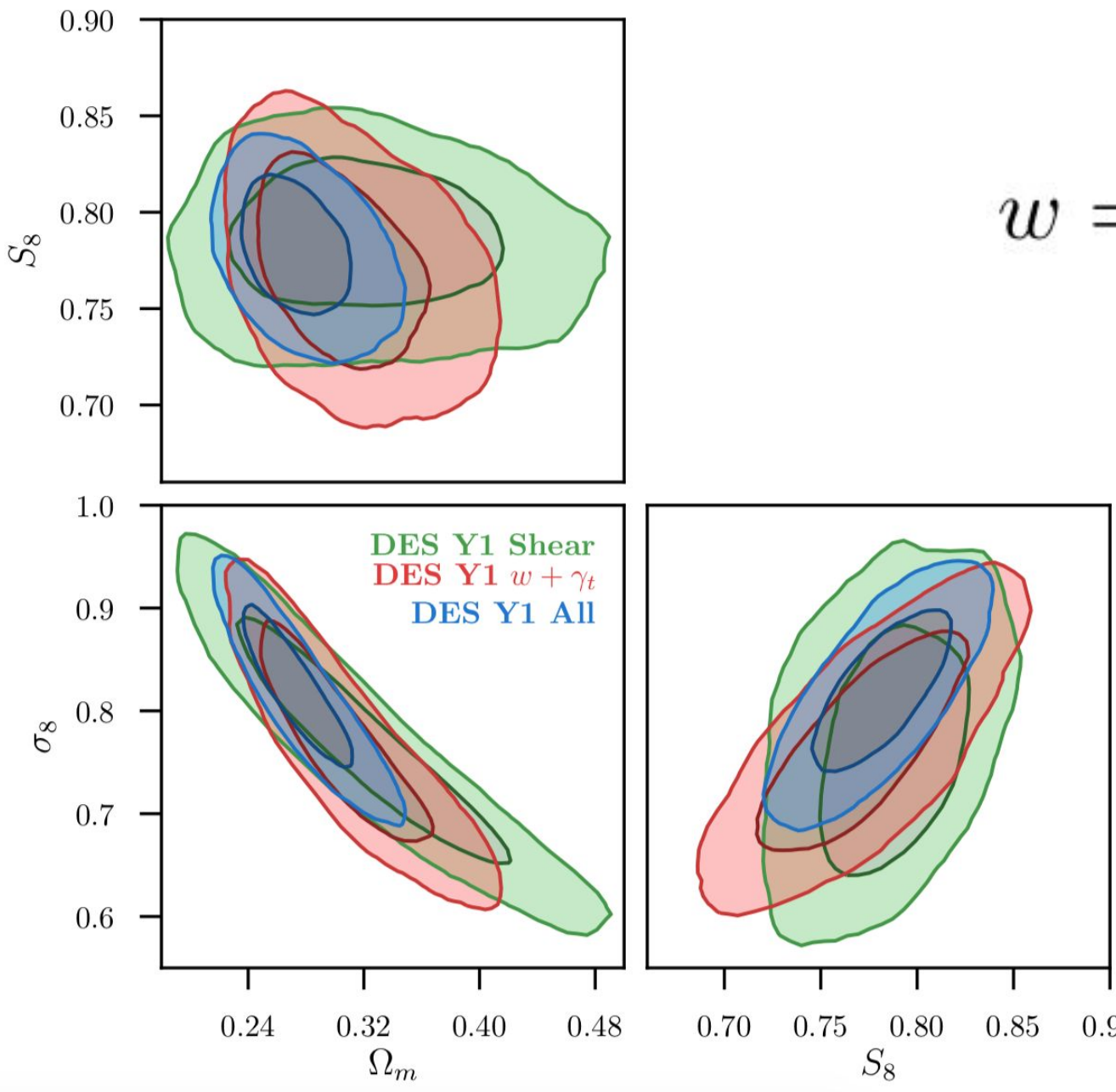

$$
w=-1.00_{-0.05}^{+0.04}
$$

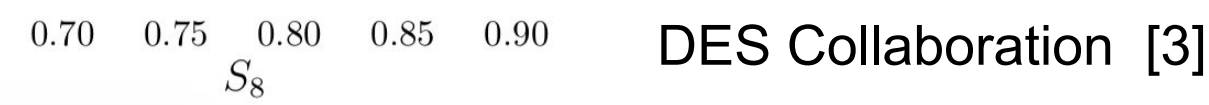




\section{Balrog}

- "digging too deeply and too greedily into data"

- Understand systematic biases $\rightarrow$ useful for all DES working groups

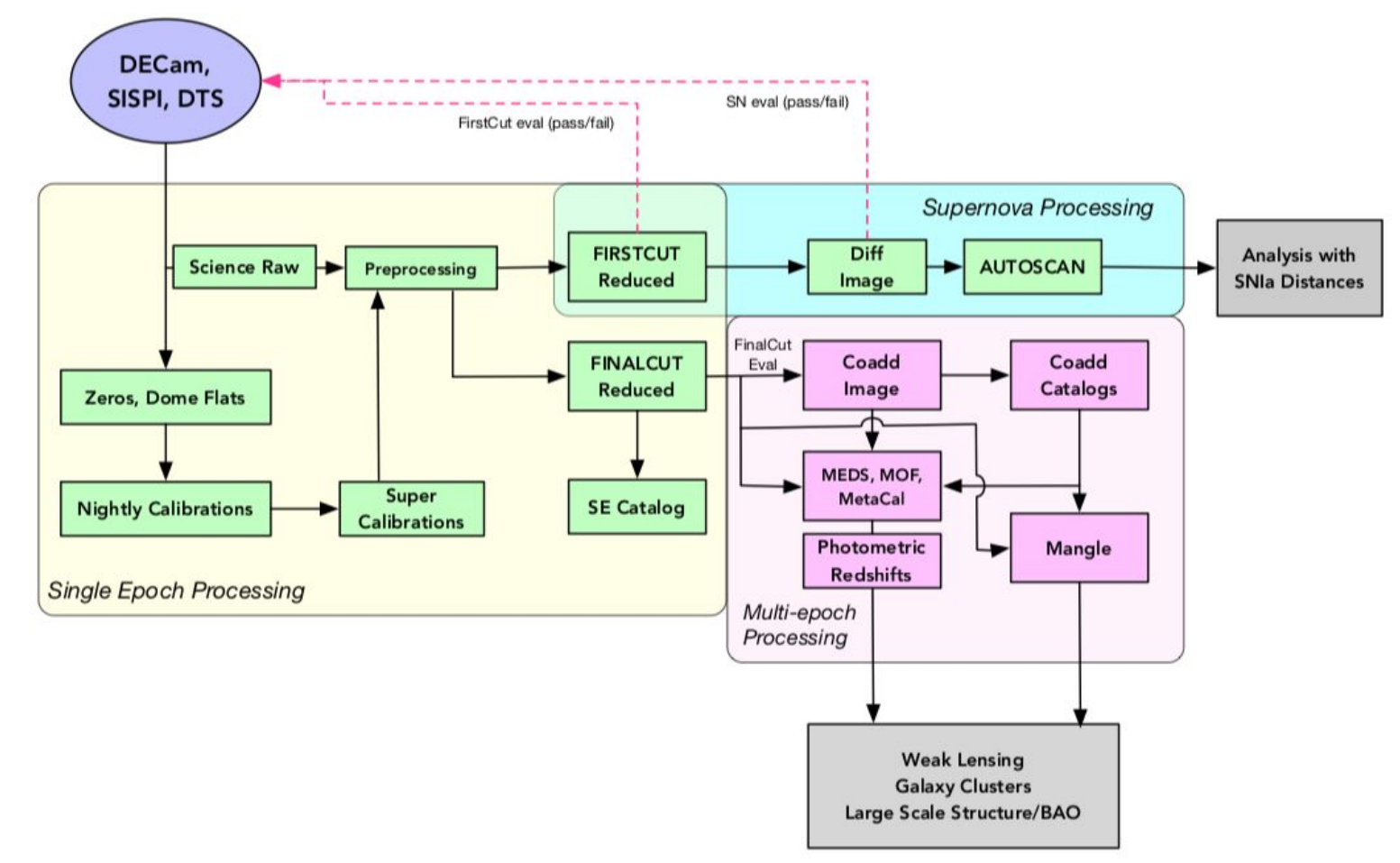

DESDM pipeline schematic [4] 


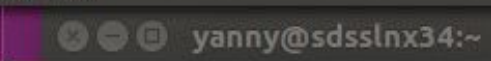

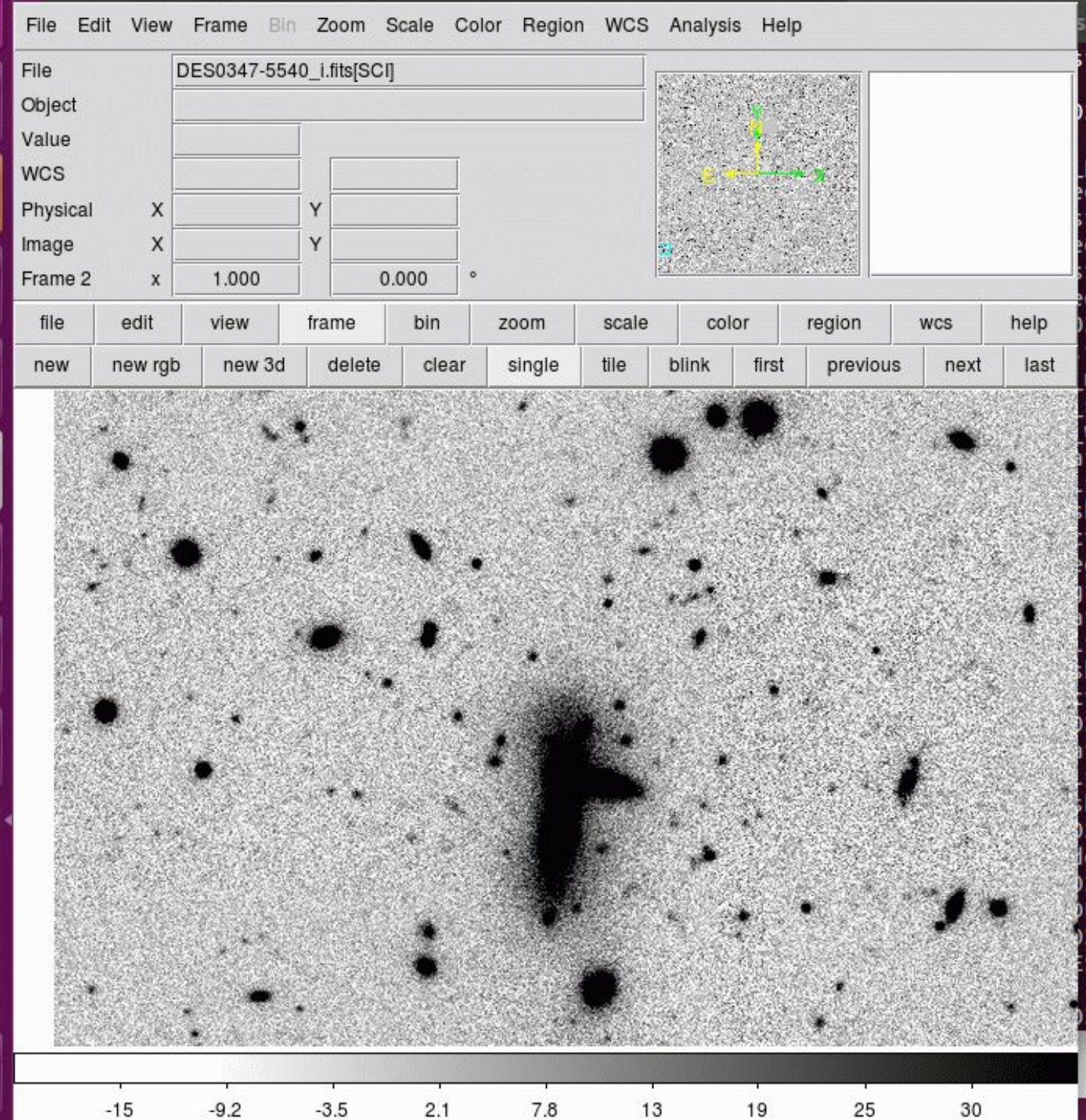

\section{Balrog injections}




\section{Q-O yanny@sdssinx34:}

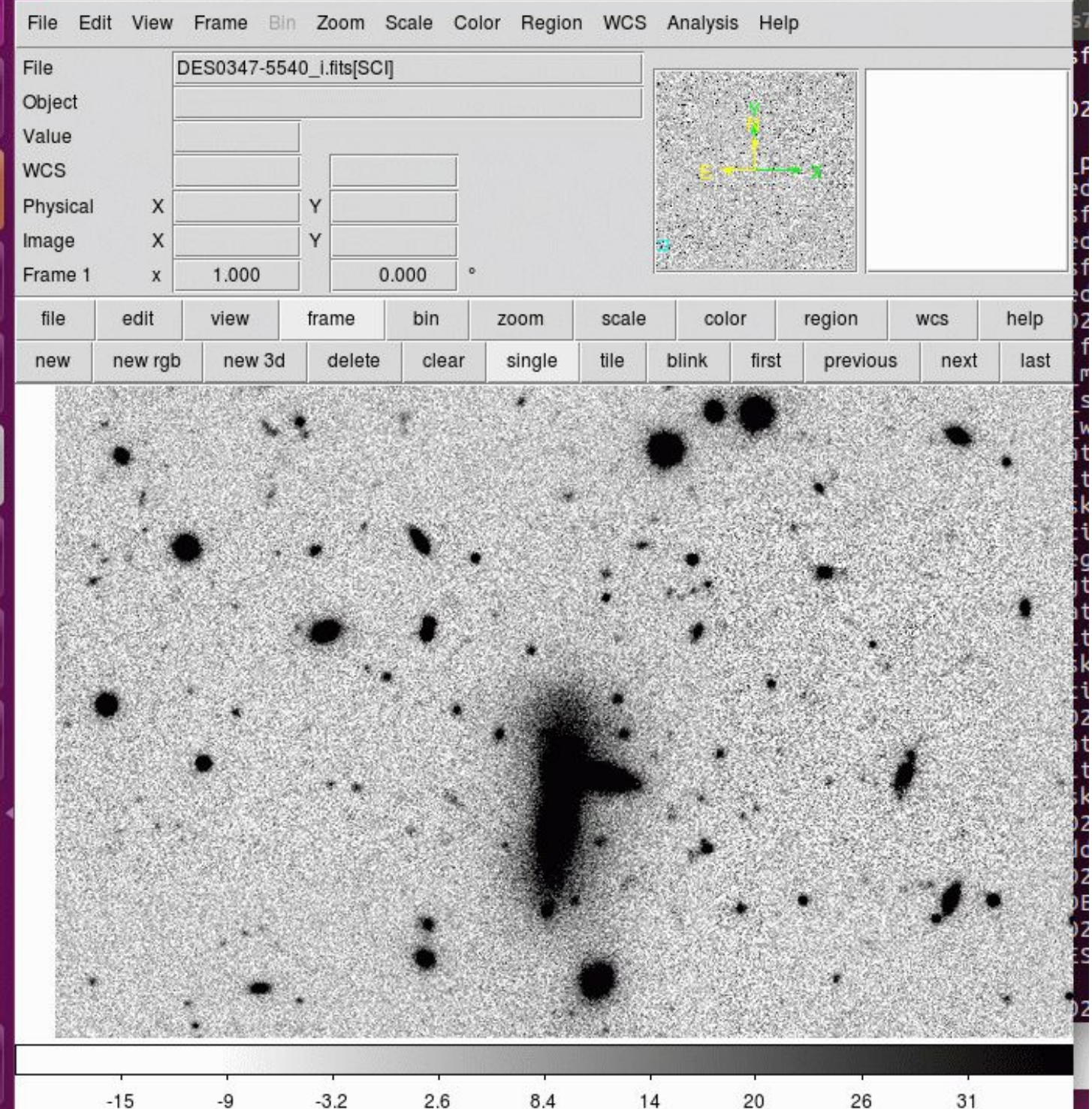




\section{(8) - ㄱanny@sdssinx34:}

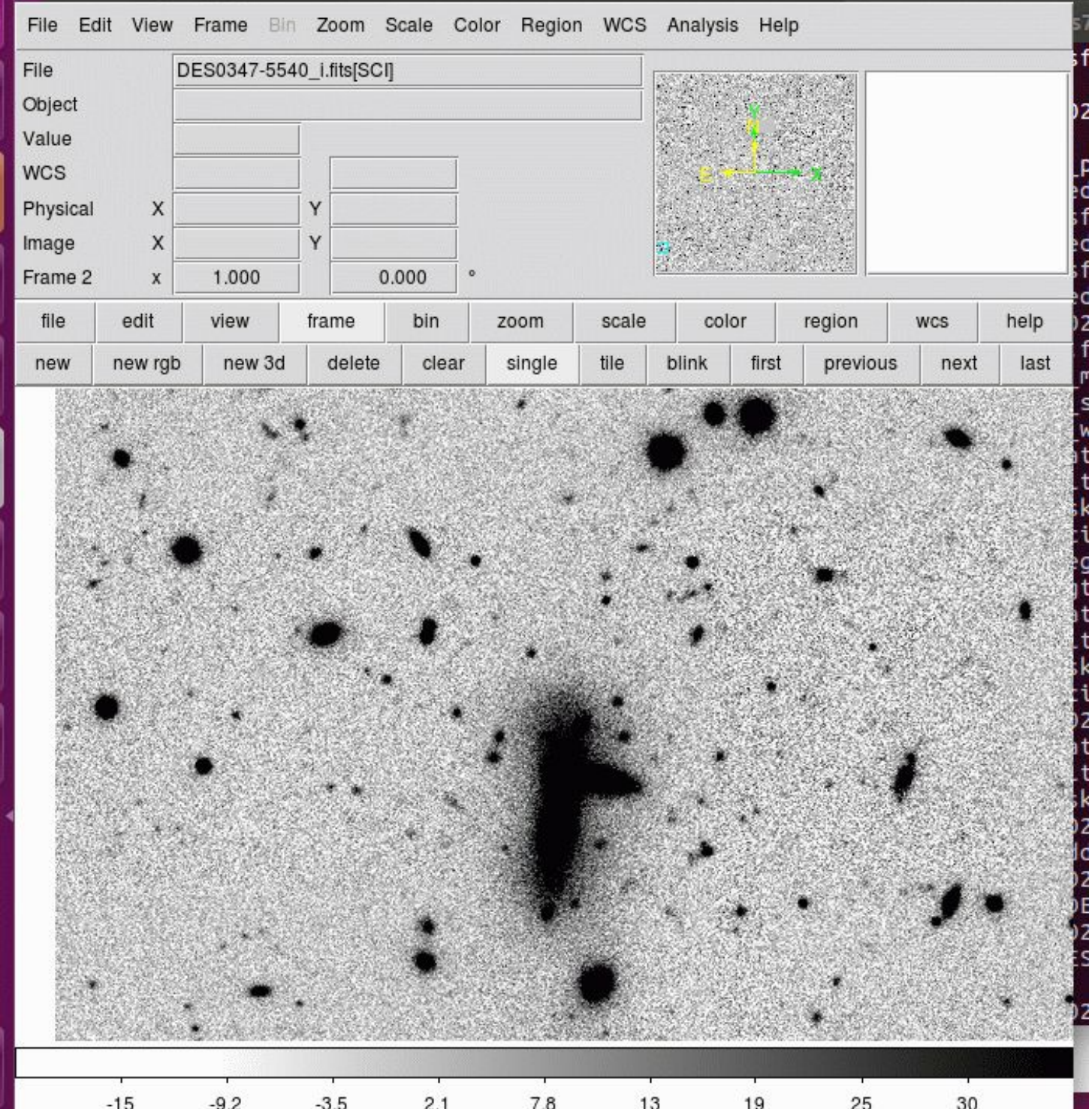




\section{Balrog validation}

- Inject fake objects onto real images. Compare truth, real, and injected catalogs

- Agreement between real \& injected and truth \& injected?

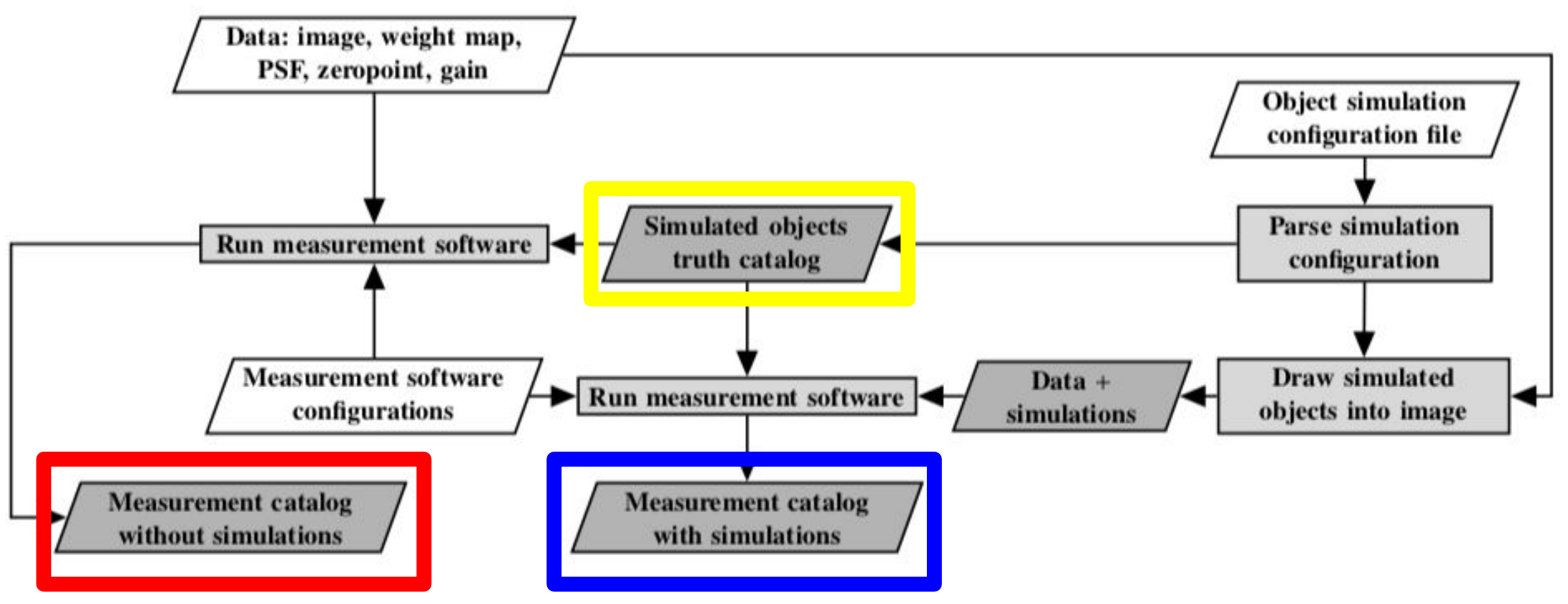

Balrog overview [5] 


\section{Summary}

- Tons of science is done with DES data (much more than discussed here)

- Balrog has the potential to help DES utilize more of its data

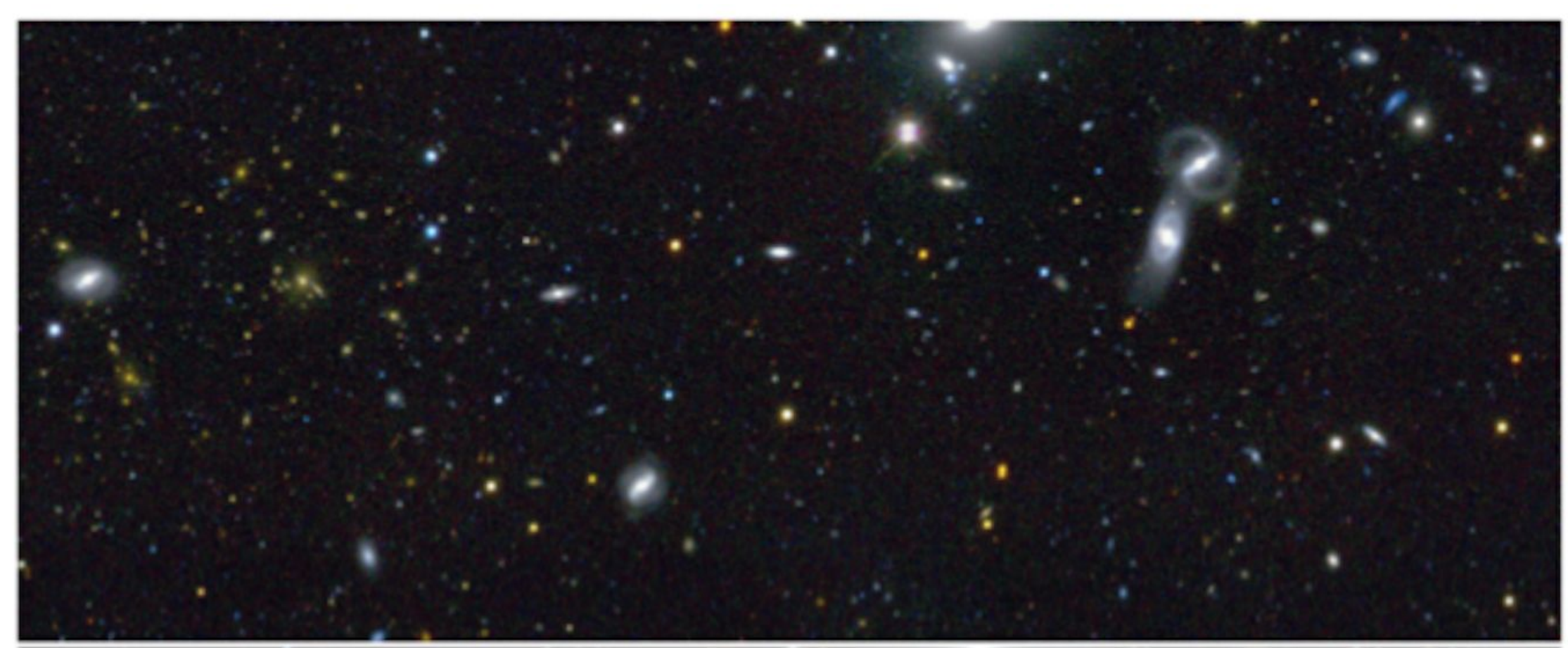




\section{Dark Energy Survey Working Groups}

- Galaxy Clusters

- Supernovae

- Weak Lensing

- Theory and Combined Probes

- Milky Way

- Strong Lensing
- Large-Scale Structure

- Simulations

- Redshift

- Galaxy Evolution \& QSO

- Transients \& Moving Objects

- Science Release

- Science Committee 


\section{References}

[1] https://arxiv.org/abs/1801.03181

[2] https://arxiv.org/abs/1801.03097

[3] https://arxiv.org/abs/1708.01530

[4] https://arxiv.org/abs/1801.03177

[5] https://arxiv.org/abs/1507.08336

Balrog software: https://github.com/sweverett/Balrog-GalSim, https://github.com/emhuff/Balrog

Some validation tests: https://github.com/spletts/BalVal 
Extra Slides...

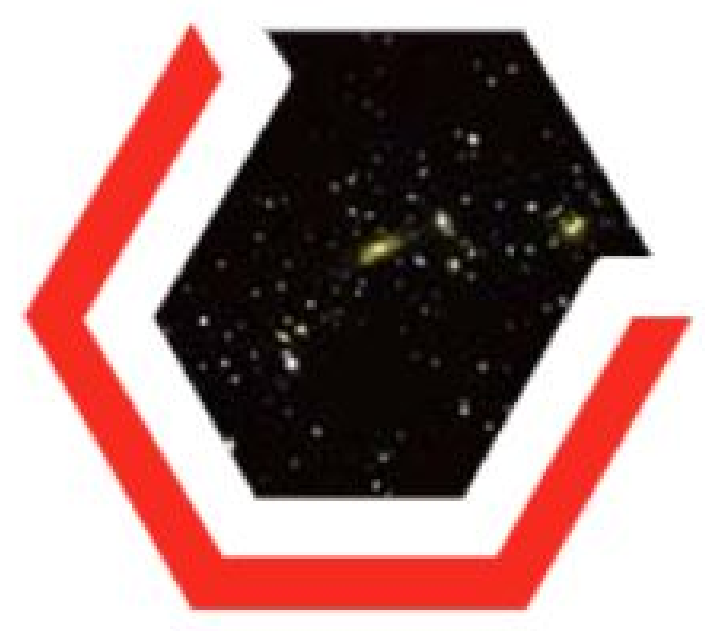




\section{Probes}

- Supernovae

- Galaxy Clusters

- Gravitational Lensing

- Baryon Acoustic Oscillations 


\section{Dark Energy Camera}

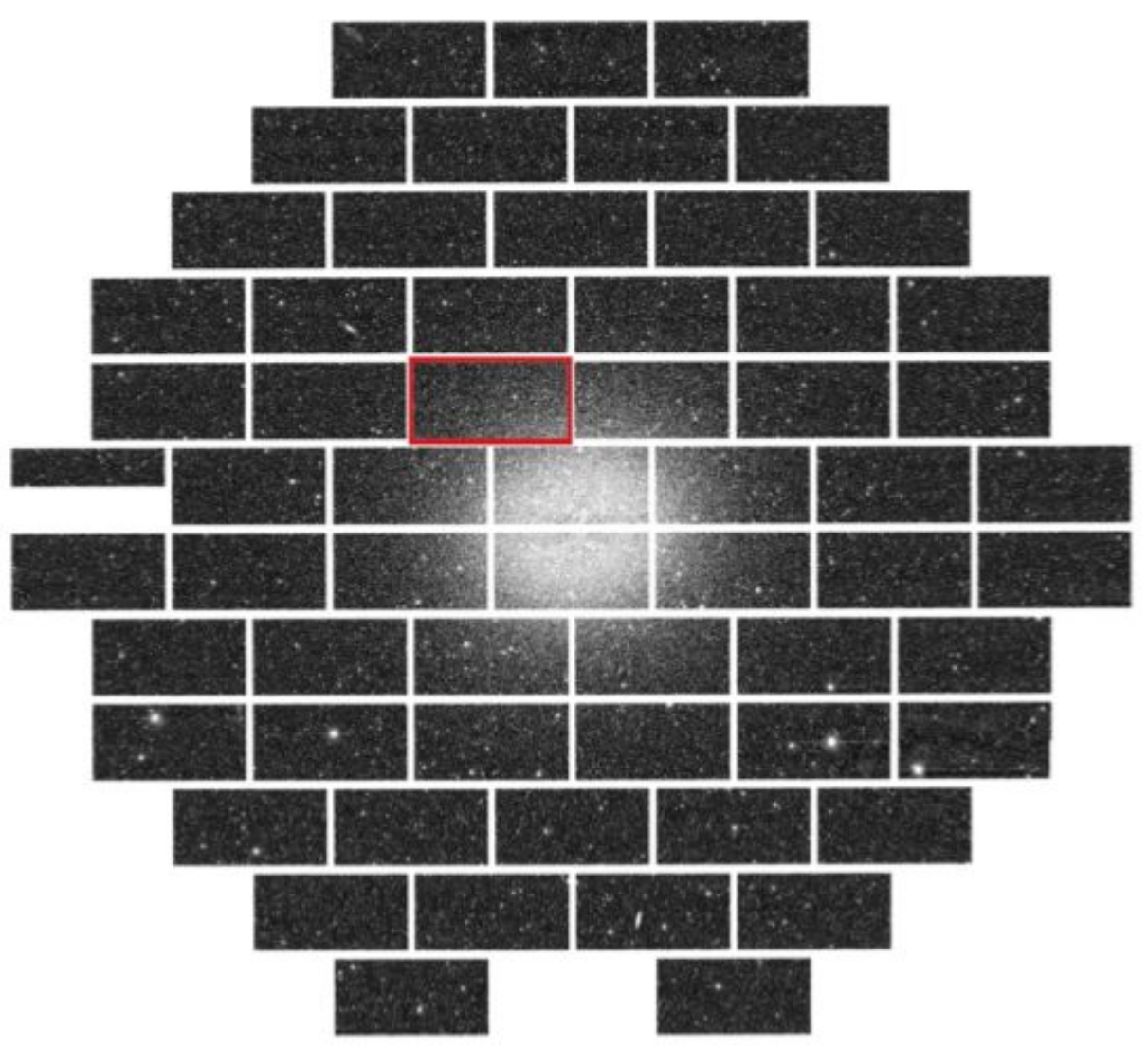

Camera image [1] 


\section{DES Y1 comparison to Planck}

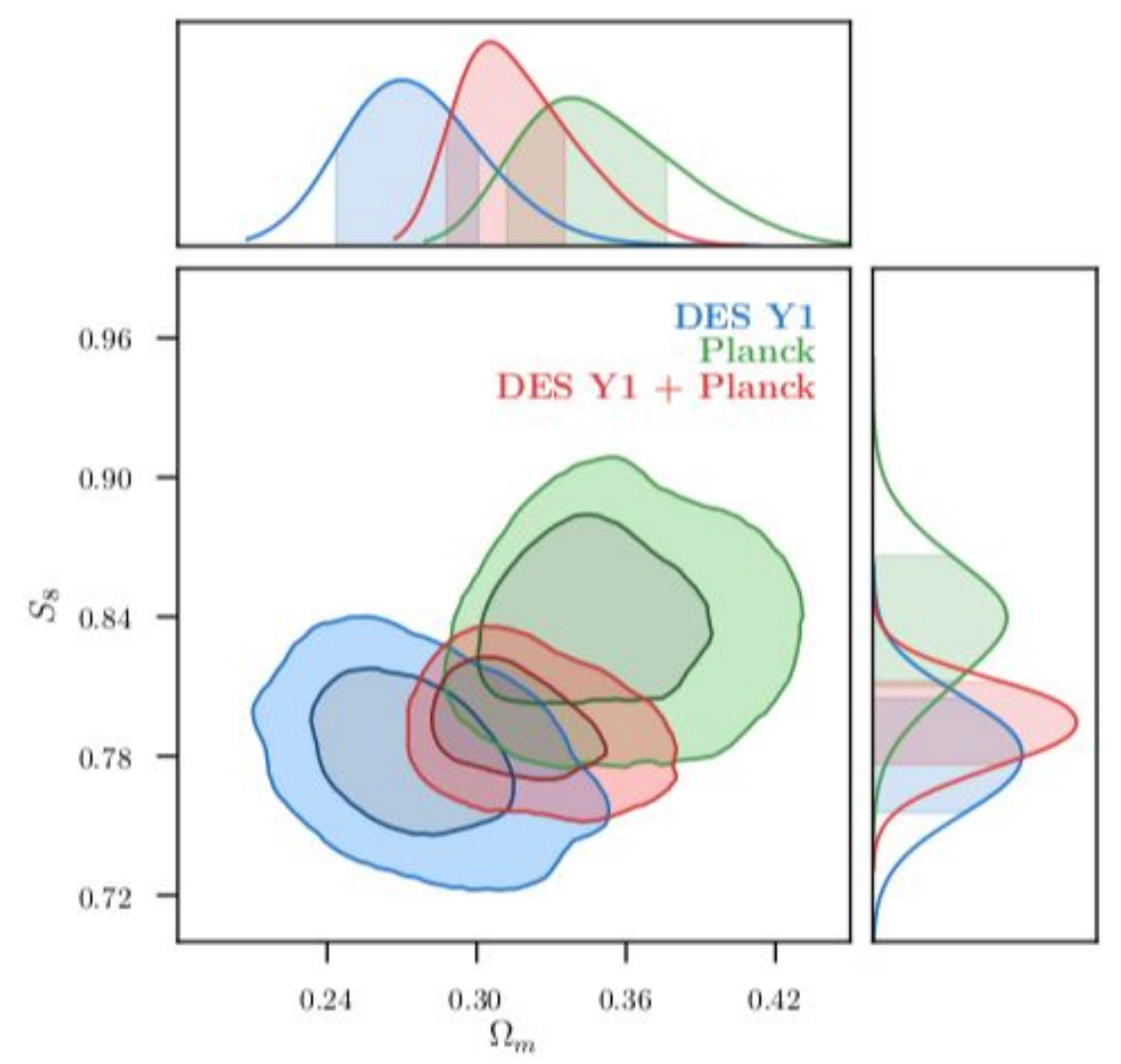


Kilonova

GW170817

DECam observation

(0.5- 1.5 days post merger)

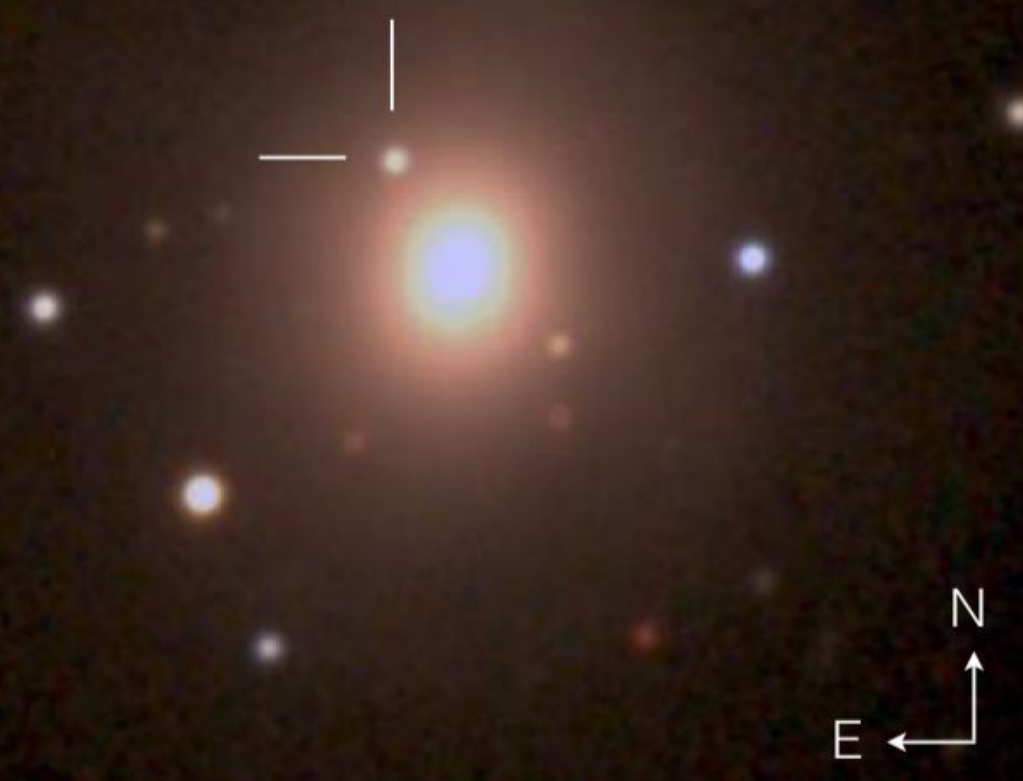

https://arxiv.org/abs/1710.05459 


\section{Science Verification Balrog Results}

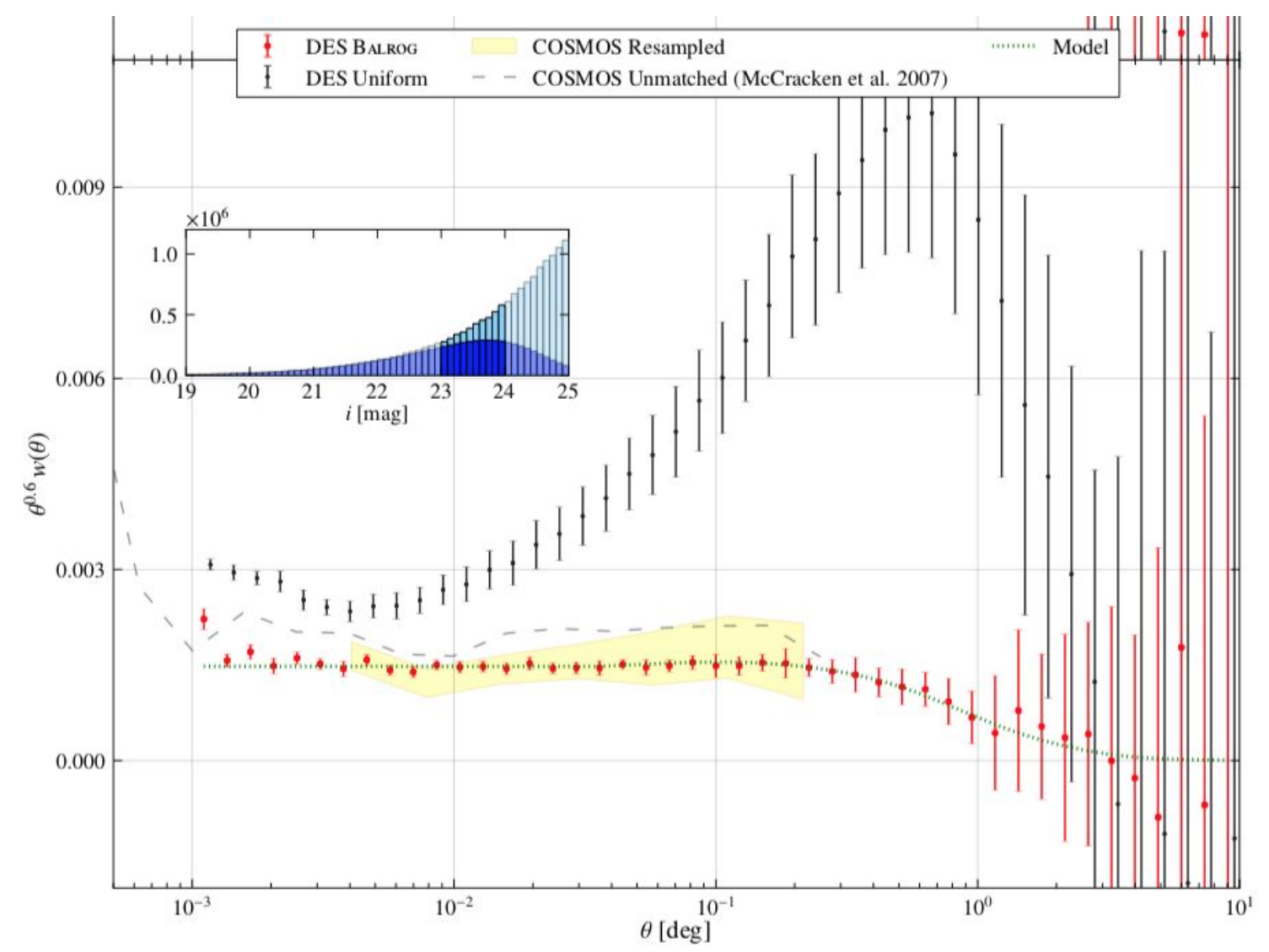

Reference [] 


\section{Validating Balrog -- Flux}

10\% Inj Gal Truth Cat \& 10\% Inj SOF Cat. Tile: DES0220-0207. Realization: 0.

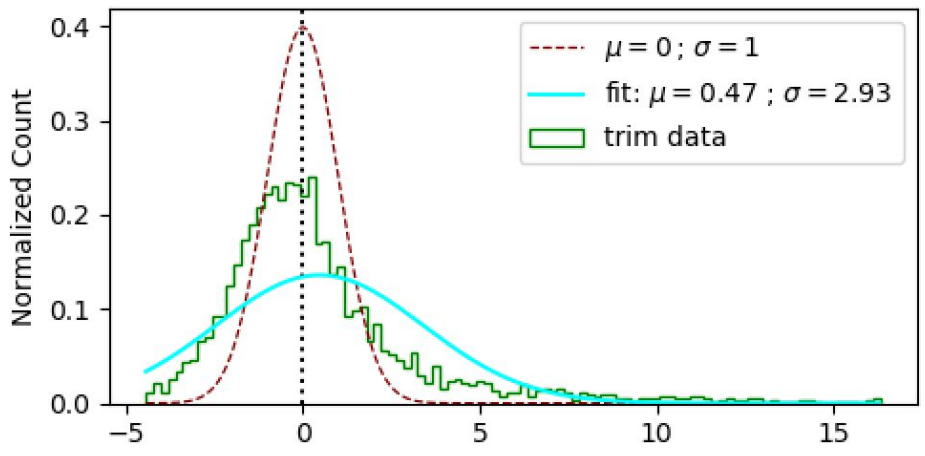

(10\%_inj_cm_flux_g meas - true) $/ \sigma_{\text {filux }}$ meas

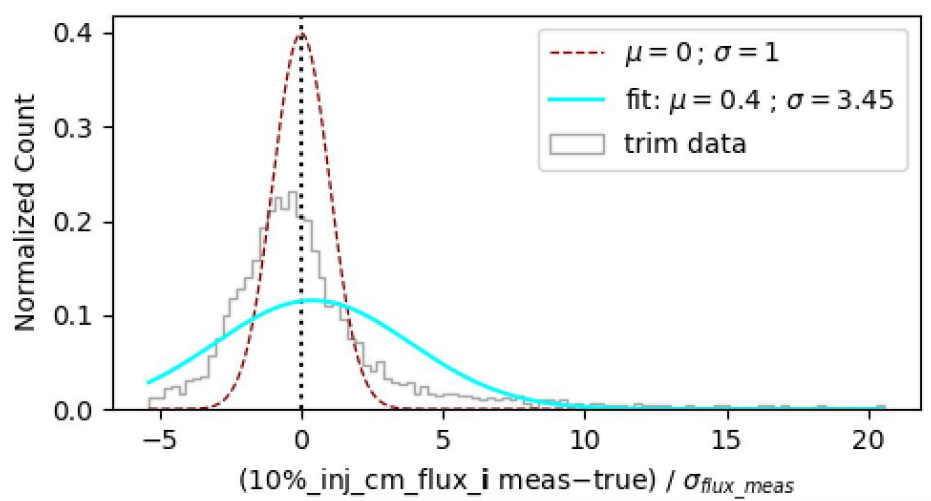

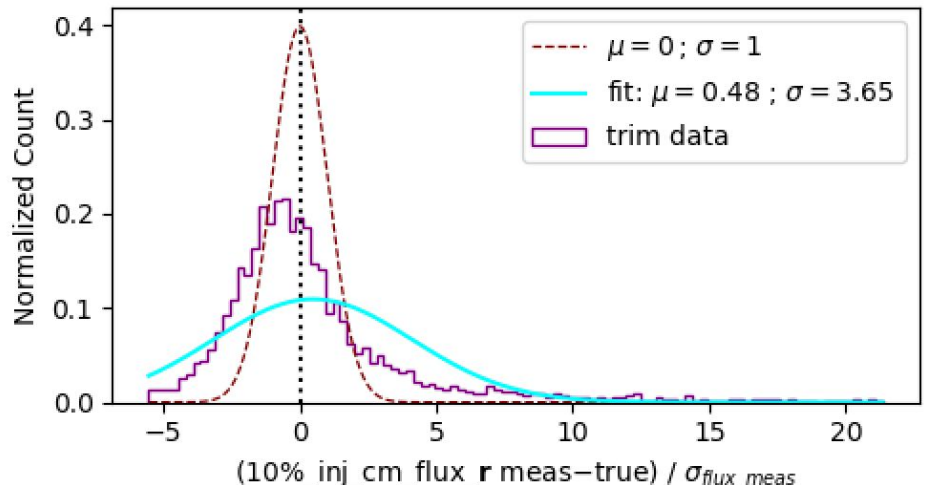

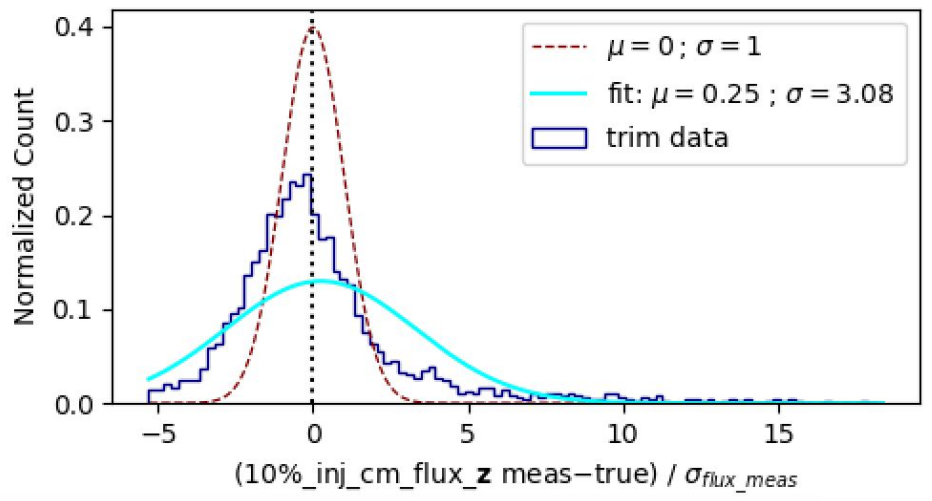




\section{Validating Balrog -- Magnitude}

20\% Inj Gal Truth Cat \& 20\% Inj MOF Cat. Tile: DES0102-4914. Realization: 0. Recovered (with flags): 83.17\%
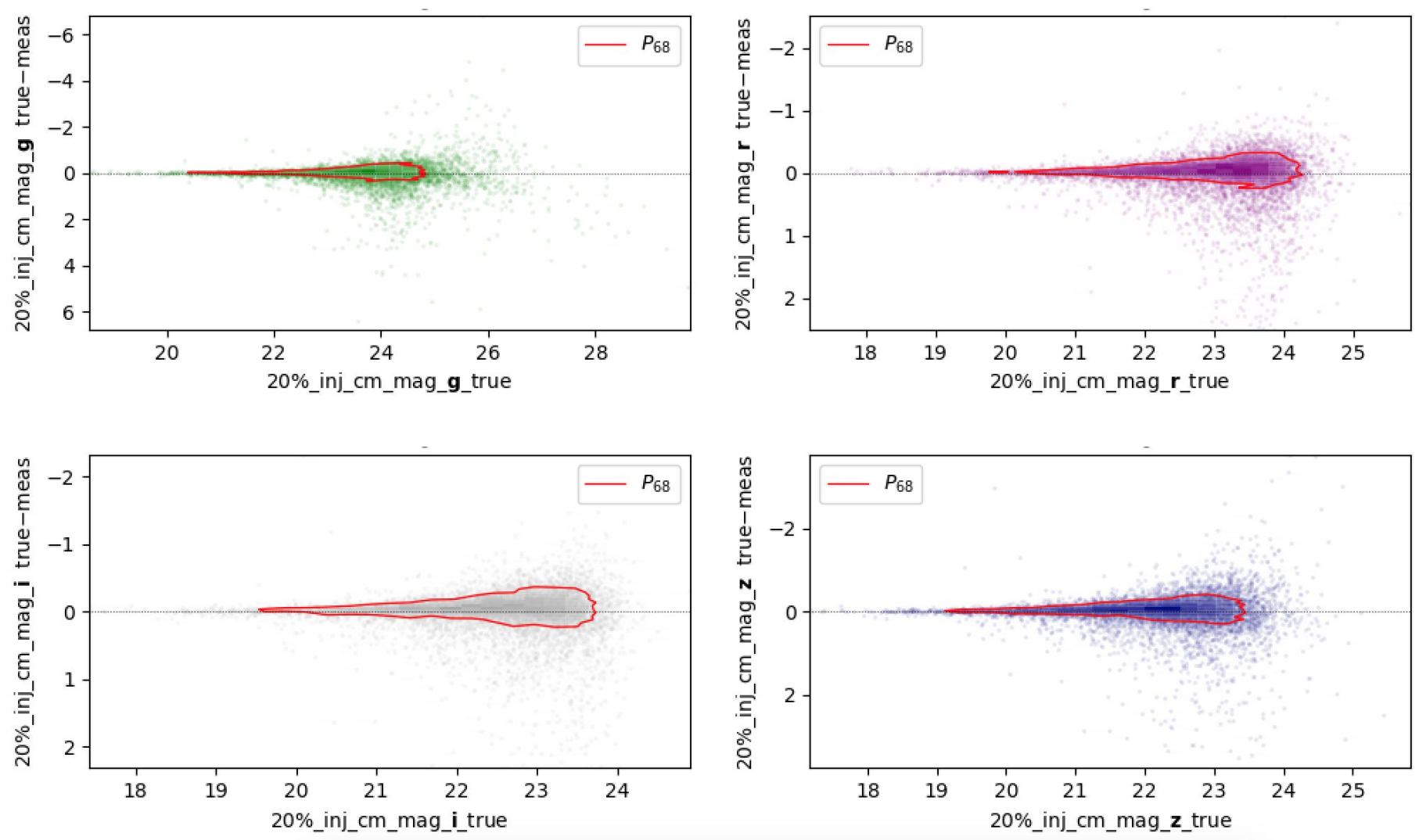


\section{Validating Balrog -- Completeness}

Inj Gal Truth Cat \& Inj MOF Cat. Tile: DES0236+0001. Realization: 0.

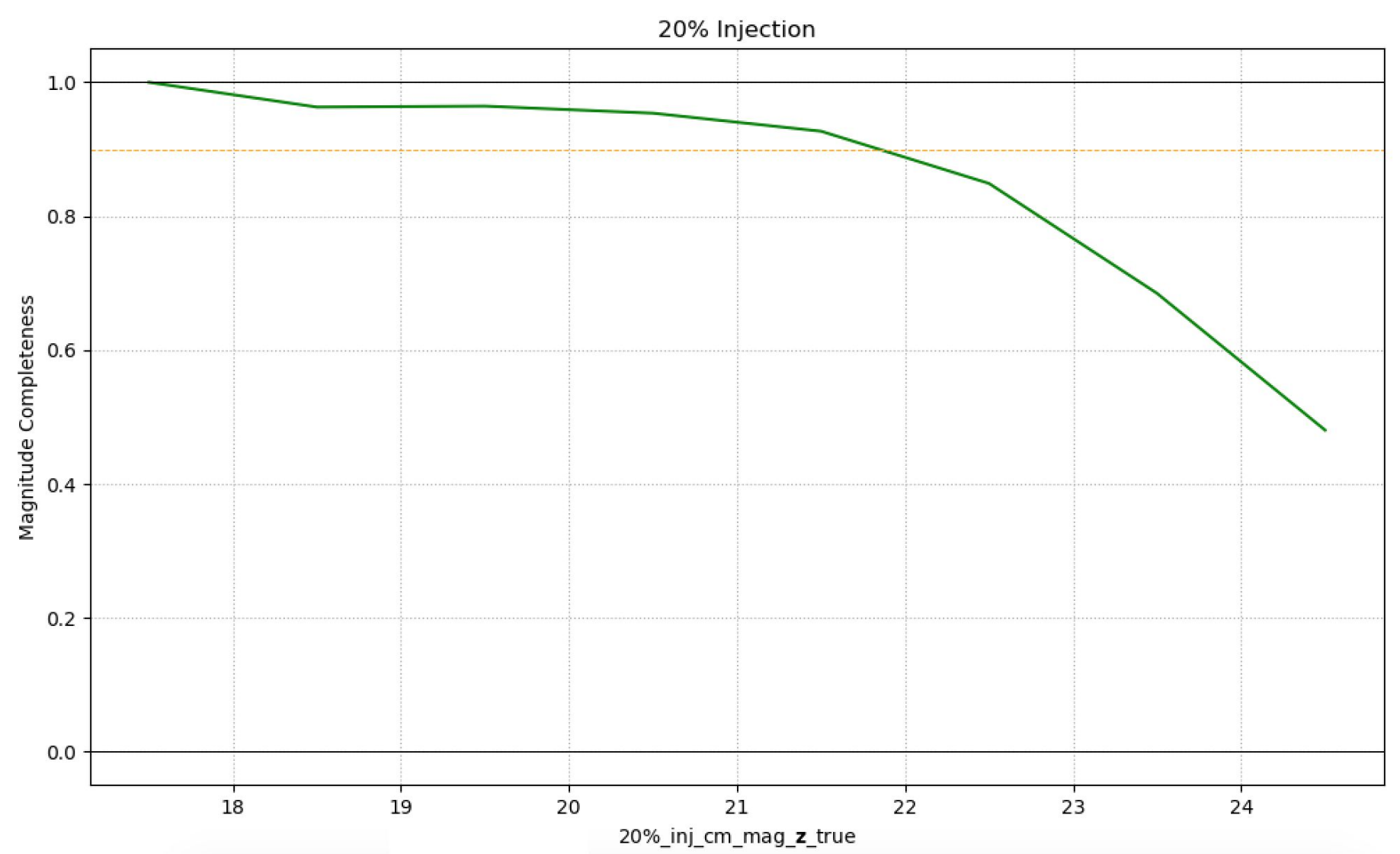




\section{Validating Balrog -- Color}

20\% Inj Gal Truth Cat \& 20\% Inj MOF Cat. Tile: DES0102-4914. Realization: 0.
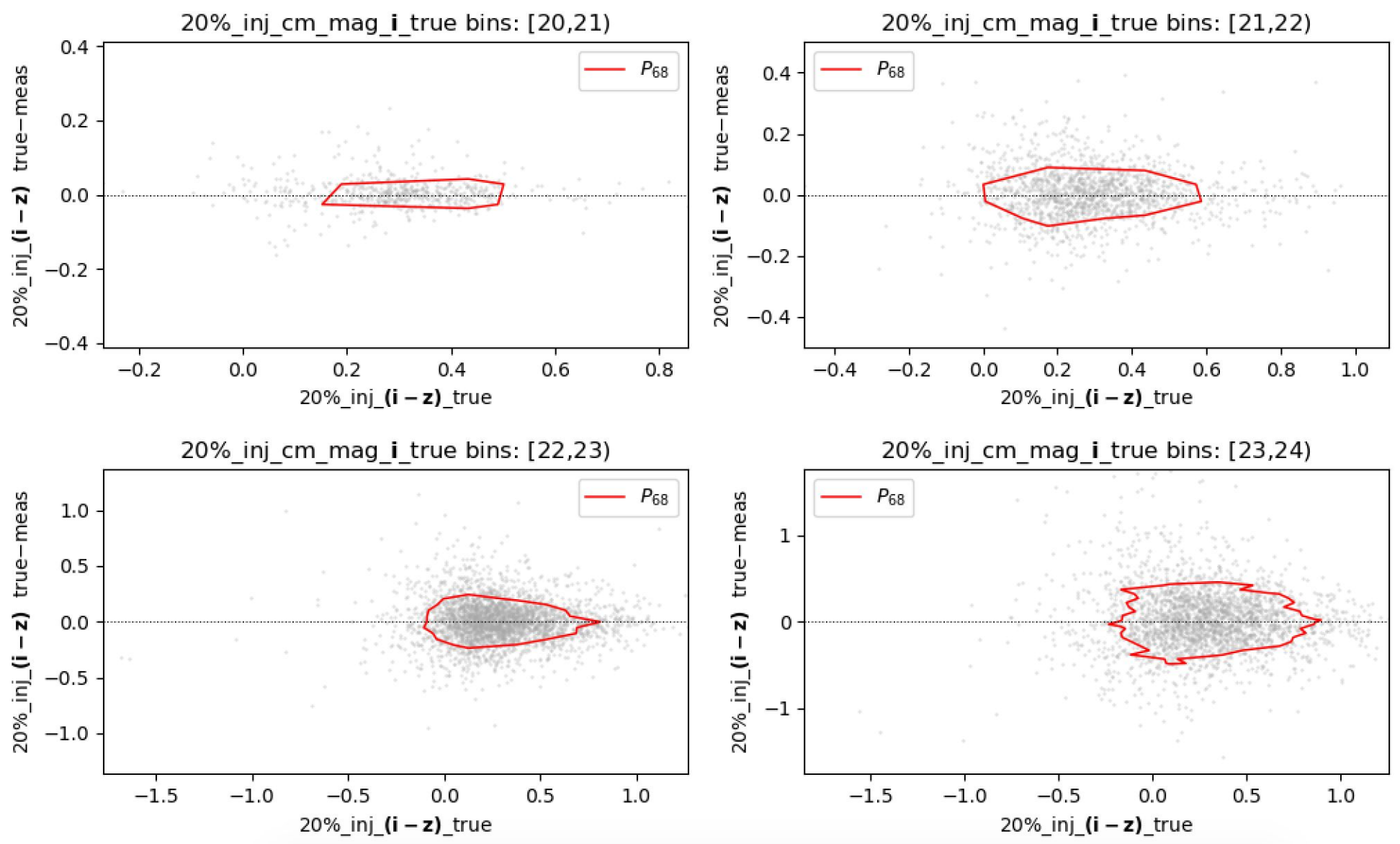